\title{
4. Wallowing in Dissonance
}

\author{
The Attractiveness of Impossible Puzzle Films ${ }^{1}$
}

Miklós Kiss and Steven Willemsen

If you're not confused, you're not paying attention.

- Tom Peters

There has been no shortage of attention in film studies regarding the current trend of complex stories and storytelling. Discussing the increasing prominence of perplexing narrative forms both in popular cinema and serialized television, which appears to have emerged from the mid-199os onward, scholars have spoken of "complex narratives" (e.g., Staiger 2006; Simons 2008; Mittell 2015), "puzzle films" (Panek 2006; Buckland 2009, 2014a), "mind-game films" (Elsaesser 2009, 2017) and "modular" (Cameron 2008), "mind-tricking” (Klecker 2013), or "multiform" narratives (Campora 2014). These diverse labels have been used to cover not only a wide range of films (from cult hits and mainstream blockbusters to international and historical art cinema), but have also been accompanied by a variety of approaches. Scholars have used narratological approaches to provide typologies and taxonomies of various complex films, have examined the (film-) philosophical implications of these new narratives, or have focused on the cultural, sociological, industrial, technological, or media-archaeological contexts from which the trend has emerged.

In our monograph, Impossible Puzzle Films: A Cognitive Approach to Contemporary Complex Cinema, we proposed yet another angle, aiming for an in-depth understanding of the effects and experiences of narrative complexity in contemporary cinema. We offered a "cognitive reconceptualisation" of story and storytelling complexity in film by analyzing how different types of complex movies evoke different kinds and degrees of cognitive puzzlement in their viewers, leading to various viewing effects and experiences. Our inquiry led us to further questions, such as what kinds of interpretive responses complex film narratives evoke and encourage, and how different films have used different modes and degrees of complexity (from moderately complex "puzzle" and "twist" films to highly disruptive and excessively complex story structures, in both popular film and art cinema). This approach singled out a distinct set of movies that we labeled 
"impossible puzzle films": popular films that evoke pervasively confusing viewing experiences, undermining narrative comprehension by means of various complicating storytelling techniques and the eliciting of dissonant cognitions (Kiss and Willemsen 2017, 59). We argued that films, such as Mulholland Drive (2001), Primer (2004), Triangle (2009), or Arrival (2016) feature notable degrees of narrative confusion, and employ (counter) strategies by means of which they strive to keep viewers interested and immersed in their stories' challenges and mysteries.

When trying to understand the nature of the viewing experiences that complex narratives such as impossible puzzle films provide, one question lurks constantly around the corner: Why would anyone be interested in confusing stories? After all, why would viewers spend hours attempting to solve potentially unsolvable puzzles? What pleasure could we take in fictional stories that are manifestly designed to be excessively complex?

In the following excerpt from the final chapter of our book, we freely ponder this question: What makes highly complex stories attractive or at least engaging for (some) viewers? It is not our aim to provide definitive answers. Thinking about complex film narratives' potential for engagement or attractiveness implies other important issues that can be rather thorny (such as why people engage with art and fiction in the first place). Queries of this kind also generally resist easy or univocal explanations. Moreover, what people draw from these particular films is likely to vary significantly according to their individual film and media literacy, personal history, preferences, competences, and attitudes. Undoubtedly, there is also a significant number of viewers who do not like this type of cinema, or with whom perplexing stories simply do not resonate at all. Nevertheless, these caveats do not make the question irrelevant - on the contrary, understanding what draws some people to complex stories is a fundamental part of understanding these films themselves, both in terms of the viewing experiences of those who watch them, and as a phenomenon in contemporary audiovisual culture. Therefore, to open up the discussion and disclose further perspectives, we will devote this contribution to contemplating the possible attractiveness of complexity, inspired and informed by the observations we have made in our earlier studies on cinematic narrative complexity (Willemsen 2018; Ros and Kiss 2018; Kiss and Willemsen 2017; Willemsen and Kiss 2017; Coëgnarts et al. 2016; Kiss 2012, 2013). It is an attempt to look beyond our usual theoretical frameworks, loosening the scientific rigor, and taking a stance that is, admittedly, a speculative one.

Most of the popular "puzzle films" found in contemporary cinema can, in many ways, still be said to provide the type of gratifications that are 
commonly attributed to classical narrative film. From a cognitive and affective perspective, Nitzan Ben Shaul characterizes the attractiveness of classical narrative cinema as follows:

It seems that the challenging of the viewers' cognitive faculties in a manner that satisfyingly lets them construct out of the movies' compelling audiovisual flow a coherent story that leads to closure, along with the attendant arousal, regulation, and control of tension, mostly through suspense strategies, are the sine qua non components that account for the popularity of movies. $(2012,25)$

But whereas many popular "puzzle films" restrict their complexity to moderate and motivated forms (Willemsen and Kiss 2017, 5), encouraging and, ultimately, rewarding viewers' intensified narrativization efforts with an attainable solution or comprehension (Kiss and Willemsen 2017, 56), our previous theorizing also proposed that other films such as the ones that we have called "impossible puzzle films" offer a more excessive complexity that frustrates viewers' narrativizing efforts more strongly, and are thus likely to offer different viewing pleasures. It is reasonable to assume that the more complex and confusing a film's narrative, the less its enjoyment will correspond to the qualities usually associated with conventionally realist and canonical "classical narratives" (e.g., immersion, identification, empathy, the arousal of emotions, and the satisfaction of closure). Films that present "impossible puzzles," apparently deny viewers much of this satisfaction. Although films, such as MULHOLland Drive or DonNie DARKo (2001) still involve classical narrative patterns and engaging affects, such as suspense and tension, they do not allow viewers clear-cut solutions to well-framed problems, and often deny narrative closure. Rather, impossible puzzle films are dissonant, ambiguous and open-ended, and may even leave viewers searching for the story; some even appear not to allow the construction of any coherent narrative chain of events. Simply put, these films are confusing - a state of mind that, arguably, most people under most circumstances would prefer to avoid. However, what appears to be an undesirable sensation in real life might be an appealing experience in mediated art; impossible puzzle films, just like perplexing and dissonant art films (think of postwar modernist art cinema), have attracted a considerable audience and critical acclaim. The question as to what underlies the fascination with such films thus becomes a rather intriguing one; it seems that complexity in a story can also entail a distinct appeal of its own. While working on our book, we accumulated some ideas and hunches for potential reasons for the 
attractiveness of cognitively dissonant and highly complex stories. Below, we will share eight of these ideas in the form of explorative hypotheses. No rigid factuality should be ascribed to these - they are not "claims" as such; rather, we hope that our reader will feel invited to think along, to bring in his or her own knowledge and experiences, and to reflect further on the possible pleasures and functions of this particular type of cinematic story.

\section{Hermeneutic Play and Interpretive Multiplicity}

One unique aspect of engaging with highly complex or impossible puzzle narratives could lie in the peculiar meaning-making activities that they allow. In a previous discussion of the possible interpretive responses to dissonant stories, we noted that they can evoke what we call hermeneutic play through repeated frame-switching (Kiss and Willemsen 2017, 130-139). Impossible puzzle films do not allow a single interpretive resolution to achieve full closure; rather, they appear to be designed to keep viewers in a loop of sense-making. In so doing, they evoke a perpetual sense of "cognitive dissonance"2 that encourages an enduring search for a satisfying resolution or a clear meaningfulness. This invites viewers to repeatedly try out different interpretations, frames of knowledge, analytical strategies, and critical competences, without necessarily settling on a single outcome. This prolonged interpretive quest, we hypothesize, can maintain a distinct interpretive multiplicity that viewers may appreciate for various reasons.

First of all, this lack of closure and interpretive hierarchy may be deemed liberating. In terms of engaging with fiction, impossible puzzle films offer an appeasing alternative to the closed, teleological cause-and-effect logic of classical film narratives. ${ }^{3}$ They refuse to adhere to the singular logic and typical closure that characterizes the vast majority of classical narratives with which contemporary audiovisual culture is saturated. Highly complex stories that challenge (but do not entirely break with) this familiar mode of classical narration may thus simply be attractive for their novelty, offering a refreshing variation on the very common ways of engaging with fiction, or even selfreflexive "metafictional" pleasures. More broadly speaking, viewers may also appreciate these films' resistance to sense and meaning-making as a triumph over reason and order at large. For instance, one frequently heard argument is that highly complex film narratives form a critique of the Enlightenment values that determine much of the modern scientific worldview (e.g., Panek 2006, 67). A work's noncompliance with being rationally contained can be appreciated as liberation from modern Western scientism, or from the 
cultural dominance of qualities, such as objectivity, logic, clarity, purposefulness, predictability, agency, and explanation. In addition, viewers may value complex classical narratives for their emancipation of alternative qualities, such as subjectivity, irregularity, contingency, unpredictability, uncertainties, pathologies, and ambiguity. Indeed, such alternative value-attributions need not be exclusive to "highbrow" art cinema. Furthermore, some individuals may simply take pleasure in being overwhelmed by an artwork that surpasses reason and cerebral comprehension. One may simply enjoy the sensation of perplexity that such stories evoke, finding pleasure in the dazed states of nonunderstanding, or in feeling the affective, nonconceptual sensations afforded by a narrative that eludes cerebral comprehension. Arguably, the quality of open-endedness in interpretation is generally also something that is appreciated in our cultural apprehension of artworks. After all, artworks that cannot be contained or exhausted in a single reading are generally held in high esteem (in many forms of art criticism, or in the canons) where such interpretive multistability is often considered an artistic asset that signals a work's depth or durability.

Secondly, viewers may also connect these qualities of interpretive multiplicity to mimetic expressivity - that is, they may see the complexity as mirroring aspects of the world in which we live, or the ways in which we experience it. Some critics have argued that complex, unsolvable narratives reflect the decentralized or diffuse postmodern culture, or the complexity of contemporary socioeconomic problems. ${ }^{4}$ It is assumed that there are viewers who feel that artworks that evoke high complexity, dissonance, or ambiguity as an effect (instead of merely depicting these conditions in their stories) do a better job at representing the inherent complexity or ambiguity of the human condition or the world around us. Moreover, films, such as MulHolland DRIVE or ENEMY (2013) may likewise be appreciated for the reason that they do justice to the complexities of the human mind, finding ways of representing the (anti)logic of dreams or the subconscious strata of the human psyche. In this sense, impossible and unresolvable puzzles may be attributed mimetic functions that can be characterized as rather existential. Jan Alber eloquently phrases such a position when pondering the appeal of "unnatural" fiction (physically, logically, or humanly impossible stories) that resists meaning-making:

At the end of the day, all examples of unnaturalness can be read as saying something about us and the world we live in. [...] For me the unnatural addresses one fundamental aspect of our being in the world: the lack of order and meaning and the difficulties of coming to terms with this 
lack. [...] The unnatural [...] reminds us of the fact that we are never fully in control of things: represented impossibilities challenge the search for order and meaning in a radical way. At the same time, however, it is of course our human predicament not just to stare into this abyss but also to try to come to terms with it. $(2016,36-37)$

This also points toward a third possible component behind the attractiveness of this type of hermeneutic play, namely training a real-world skill for dealing with interpretive multiplicity. If the everyday world is complex and characterized by a lack of clear order and meaning, then it follows that dealing with the multiplicity and multistability of different meanings forms a key aspect of dealing with that world. In connecting strategies formulated for fictional complexity to the ability to cope with real-world complexity, Ien Ang has called for the nurturing of a kind of "cultural intelligence":

Finding a language to understand [...] complexities - that is, to describe the specific ways in which things are "complex and contradictory" $[. .]-$. is a necessary step to generate the cultural intelligence with which to formulate "solutions" in terms of strategic, flexible, emergent, non-simplistic simplifications, rather than the reductionist and mechanistic thinking (informed by positivism) which still dominates much policy-making and problem-solving. (2011, 788-789)

Artworks can exercise our ability to cope with complex situations in real life by presenting complex stories or by foregrounding formal-structural complexity that requires viewers to juggle multiple, simultaneously reasonable interpretive options. The tendency of impossible puzzle films to withhold closure and unambiguous meaning can also be seen in this light. These films may, for instance, train viewers in what Reuven Tsur has labeled negative capability. Tsur quotes Keats to characterize negative capability as a competence "of being in uncertainties, Mysteries, doubts, without any irritable reaching after fact and reason" $(1975,776)$. This stands in opposition to what Tsur calls the "quest for certitude": the urge to distill singular, unambiguous meaning from an artwork and reach interpretive closure. These notions form two poles in a spectrum, ranging from the appreciation of fixedness and certitude to the valuing of lingering ambiguity and interpretive multiplicity. One may assume that a viewer's position on this spectrum will be determined by personal attributes and dispositions (cf. an individual's psychological "need for closure" - see Webster and Kruglanski 1994), and that this position is relevant in the degree to which one enjoys or values 
ambiguous artworks. Nonetheless, it can be hypothesized that repeated exposure to narrative artworks that highlight interpretive multistability may serve to train everyday "negative capability." By altering the shortcuts in an individual's meaning-making routines, repeated exposure to interpretive multiplicity may make him or her less prone to readily seeking interpretive closure.

Lastly, even if complex films do not necessarily form "cognitive playgrounds" in which viewers can train and test the meaning-making skills demanded by an increasingly complex world, then they can still be said to simply entertain skills that viewers already possess. That is, complex stories can trigger the use of certain interpretive and analytical mental competences, which viewers may enjoy exercising simply for their own sake. Following Liesbeth Korthals Altes, we could call this aesthetic pleasure Funktionslust. According to her, there seems to be a:

pleasure and interest our minds seem to take in complexity itself, admittedly in different degrees. This pleasure seems akin to what the German psychologist Karl Bühler called Funktionslust. This eloquent term refers to the pleasure taken in exercising a mental or bodily function (Bühler $1965,157)$. Such function-oriented pleasure can be observed in repetitive movements in animal and child play but also in adult behaviour, from a good physical workout to riddles or crosswords that engage the pleasure of puzzling and pattern-seeking minds. (2014, 23; our emphases)

An impossible narrative puzzle may provide viewers with a similar pleasure by entertaining their Funktionslust in repeatedly utilizing their analytical and interpretive abilities. Complexity of narrative form, Korthals Altes notes, is particularly likely to become the target of such enjoyment, as "the pleasure we may take in our skillfulness in understanding intricate form may also appear like the Funktionslust of puzzling and pattern-seeking minds" $(2014,131)$. To a degree, however, this could of course be said of aesthetic and narrative engagement in general. The idea that emerges here is akin to a more general Kantian view of aesthetics, also popular among cognitive theorists of art, which assumes that part of the gratification of art lies in the fact that it affords a free play of our cognitive-perceptual and imaginative abilities in the absence of direct purposefulness. As David Bordwell notes:

In our culture, aesthetic activity deploys such [everyday cognitive] skills for nonpractical ends. In experiencing art, instead of focusing on the pragmatic results of perception, we turn our attention to the very process 
itself. What is nonconscious in everyday mental life becomes consciously attended to. Our schemata get shaped, stretched, and transgressed; a delay in hypothesis-confirmation can be prolonged for its own sake. And like all psychological activities, aesthetic activity has long-range effects. Art may reinforce, or modify, or even assault our normal perceptual-cognitive repertoire. $(1985,32)$

In this respect, too, impossible puzzle films can be seen as having rather unique reflexive functions. Through their problematization of narrative construction, as well as through their interpretive challenges, these films can have viewers experience and reflect on their cognitive involvement in narrative construction or, more generally, on different sense-making processes (perceptual, narrative, interpretive). Engaged viewers' repeated attempts to come to terms with the inherent dissonances of these stories may afford a gratifying Funktionslust in the pattern-seeking and other puzzle-solving activities of their hermeneutic play.

\section{Orientation, Navigation, and Mapping}

Besides affording hermeneutic play, impossible puzzle films may also challenge other everyday cognitive skills and activities. One idea we wish to propose is that impossible puzzle films could provide special (embodied-) cognitive experiences by challenging one's real-life skills of orientation and navigation. Our hypothesis is that the pressure that such challenges exert on these skills might be a source of an enhanced viewer engagement. This first requires some explanation about the general function of orientation and navigation in relation to narrative fiction.

Elsewhere, we argued that real-life skills pertaining to everyday, embodied orientation and navigation are relevant to the processes involved in comprehending narrative structures (see Kiss and Willemsen 2017, 91-103; or Kiss 2013, 2015). Following previous accounts of embodied psychological and narrative continuity (Slors 1998; Menary 2008), we drew a link between the abilities of real-world orientation and navigation and analytical skills of plot segmentation in narrative comprehension. ${ }^{5}$ We hypothesized that viewers use basic spatial schemas in "mapping" narrative plot structures, for instance, through the mental projection of image schemas, or by mapping one's own familiar action patterns onto the experiential paths of the fictional characters. This claim considers the idea that viewers and readers "map" a story to be more than just a metaphor and that "mapping" is therefore 
not tied to strictly topographic dimensions. ${ }^{6}$ For instance, when viewers follow and trace stories by means of narrative plotting, mapping can involve spatial visualizations of temporal relations among events (by placing them on a mental timeline). Arguably, readers and viewers are willing to invest cognitive resources into creating mental models of narrative maps if their investment presumably contributes to their comprehension of a story. The challenges of (impossible) puzzle films seem to provide a cognitive playground that particularly encourages such mapping activity in one's narrative orientation.

Similar to real-world navigation, in fictional worlds, the absence of a clear reference point can lead to disorientation. This reference point can be characterized as the deictic center. In everyday navigation, the deictic center refers to the embodied ego-reference point from which we navigate space and monitor time (establishing dimensions, such as front, back, up, down, or before and after). When extended to narrative, the notion denotes our constructions of "where we are" in the story, referring to the constructed spatiotemporal coordinates of "here and now." In any narrative text or film, this deictic center is an essential feature of storytelling and the starting point from which we can make inferences about the film's narrative and visual markers (or a written text's grammatical indications) concerning the when, where, and who of the story.

In constructing a narrative plot, the deictic center positions the characters relative to the spatiotemporal progression of the storyline, advancing along with the unfolding narrative. This allows viewers to determine "where they are" in the story, and enables them to determine not only the "here and now" but also, for instance, what is a flashback to earlier or flash forward to upcoming events. In most narratives, the deictic center is communicated clearly, providing a backbone for the smooth integration of narrative information: we know where we are in a story and can map flashbacks, flash forwards, changes of scenes, and ellipses in relation to that point in space and time. In impossible puzzle films, however, determining a clear deictic center may become problematic, or even prove virtually impossible, as the result of palpable dissonances between cognitions or sheer lack of order. This is particularly evident with narratives that present impossible storyworlds such as parallel universe stories (that obscure the spatiotemporal hierarchy among their multiple realities), and/or use complex nonchronological storytelling structures, particularly loops (which can severely destabilize a clear determination of the "here and now" or the "beginning and end"). We hypothesize that impossible puzzle films can disorient viewers by either denying the designation of a clear deictic center, or by asking them to map the story from multiple deictic centers. 
As for the first option, many impossible puzzle films challenge orientation by hiding or obscuring the deictic center, leaving a high degree of uncertainty about the status of narrative information. It may, for instance, be left unclear as to whether scenes belong to the past, present, or future, or are a part of someone's hallucinations or dreams about the past, present, or future. One may, for instance, think of the extensive sections in MulHolland Drive in which the film delves into a mysterious variety of uncanny scenes and storylines (including those of the Hollywood director, his casting and the mobsters, the nightmare story at the Winkie's diner, the cowboy, the hitman, as well as the ongoing story of Betty and Rita). While the film spirals into these different nonchronologically organized and ambiguously focalized story paths, it becomes increasingly difficult for a viewer to establish how events relate to one another, or how scenes might be connected - either spatially, temporally, causally, or as a network. The film does not follow a single character who could have provided a navigable reference point through the succession of different scenes and settings; nor does MulHOLLAND DRIVE include other clear spatial or temporal markers by which events could be readily placed in relation to one another. Moreover, the few recurring characters, such as Betty and Rita, who could embody a focal(izing) center point around which these events revolve, seem to have slippery identities as well, which further riddles the story with contradictions and incoherency. As the film progresses, this continuous lack of a clear center of orientation, from which the story's dimensions could be mapped (for example, as past or present, or as a dream or reality) frustrates the engaged viewer's attempts to do so. The strategy of making a deictic reference point permanently elusive is arguably paramount to MulHOLLAND DrIVE's complex effects and, along with the film's highly uncanny and estranging film style, leads to a palpable sense of disorientation.

With regard to the second option, impossible puzzle films frequently present multiple (sometimes contradictory or paradoxical) deictic centers from which the plot needs to be mapped. This is particularly apparent in narratives that feature time loops and/or duplicating characters, as in Primer, Triangle, Timecrimes (2007), MiraQ (2006), or Reality (2014). In the convoluted time-travel logic of PRIMER, for instance, the multiplying - and, for the viewer, often indistinguishable - versions of the protagonists destabilize our ability to map the past, present, and future, because these versions all form different, simultaneously existing deictic centers which are active at different points on the film's timeline. As various incarnations of the protagonists coexist within a single looping structure, the film's spatiotemporal markers become increasingly dislocated. 


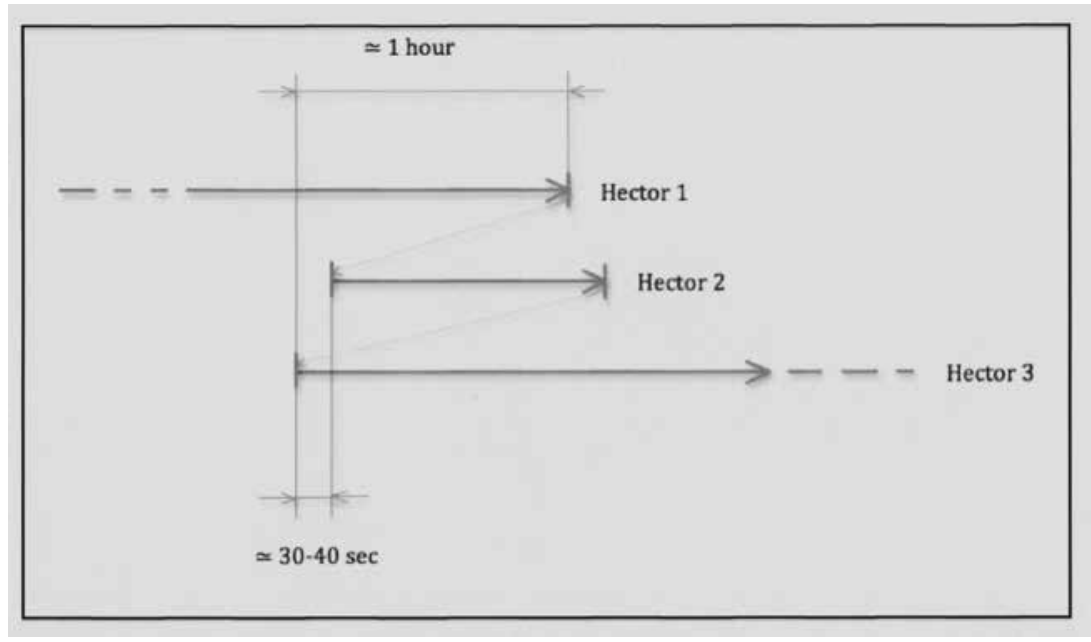

Fig. 4.1: Plot map of Nacho Vigalondo's TimeCRImes (2007), drawn by Miklós Kiss.

The effect of an intensified need for orientation in complex story comprehension can also be observed in viewers' attempts to graphically map such plots. Drawing physical maps can function as a kind of "prosthetic extension" of viewers' mental work. A physical map can unburden limited cognitive resources and working memory when coping with complex stories and plots. Visual maps of narratives might also reveal neglected clues, new semantic fields, overlooked relations and patterns, and other forms of internal logic, which otherwise could have escaped one's awareness (for example, the plot map of TIMECRIMEs reveals a simple structure behind the complex experience - Fig. 4.1). ${ }^{7}$

We would not claim that these films' challenging of deeply engrained skills of orientation and navigation is attractive in itself. However, such complexifying narrative tactics can be seen as invitational strategies that encourage heightened viewer activity, and thereby even manage to pull some viewers into playing along with the puzzle-solving games of navigational challenge, and mentally or even graphically mapping the intricate plot at hand, as the abundance of available online plot maps of complex films demonstrates. Inspiring such augmented analytical and interpretive activities, movies, especially of the complex kind, often provide models for such mapping practices themselves: from Robert Zemeckis's BACK TO THE FUTURE PArt II (1989) through Mennan Yapo's Premonition (2007) to Timecrimes, many films present pensive characters chalking diagrams or grabbing pen and paper (see Fig. 4.2 to 4.4, respectively). 


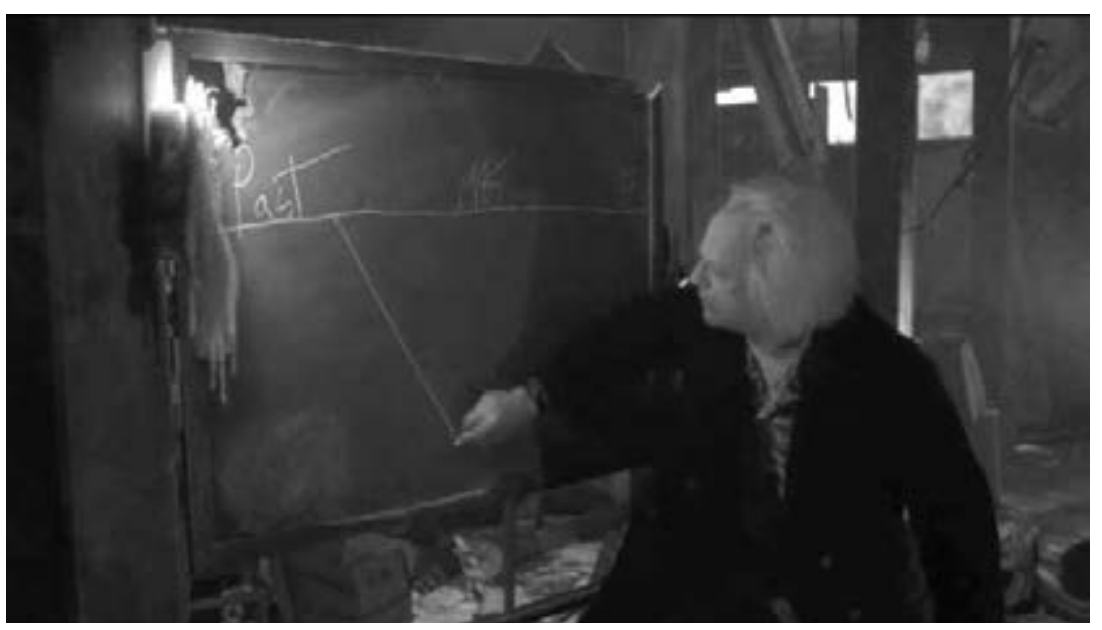

Fig. 4.2: Drawing on a blackboard, Doctor Emmett Brown (Christopher Lloyd) explains the consequences of time travel in Robert Zemeckis's Back to the Future Part II (1989).

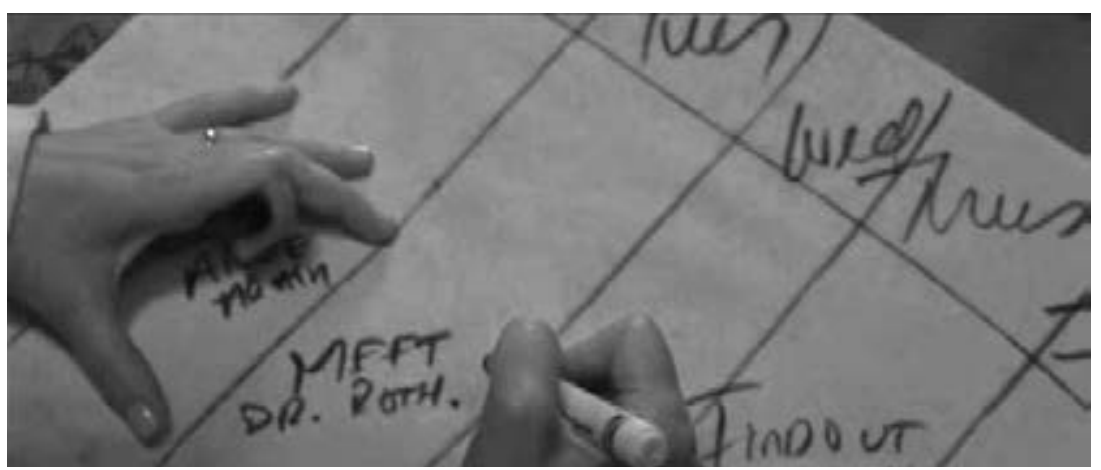

Fig. 4.3: In Mennan Yapo's Premonition (2007) Linda Hanson (Sandra Bullock) draws a calendar to be able to reconstruct a week she experiences in a nonchronological order.

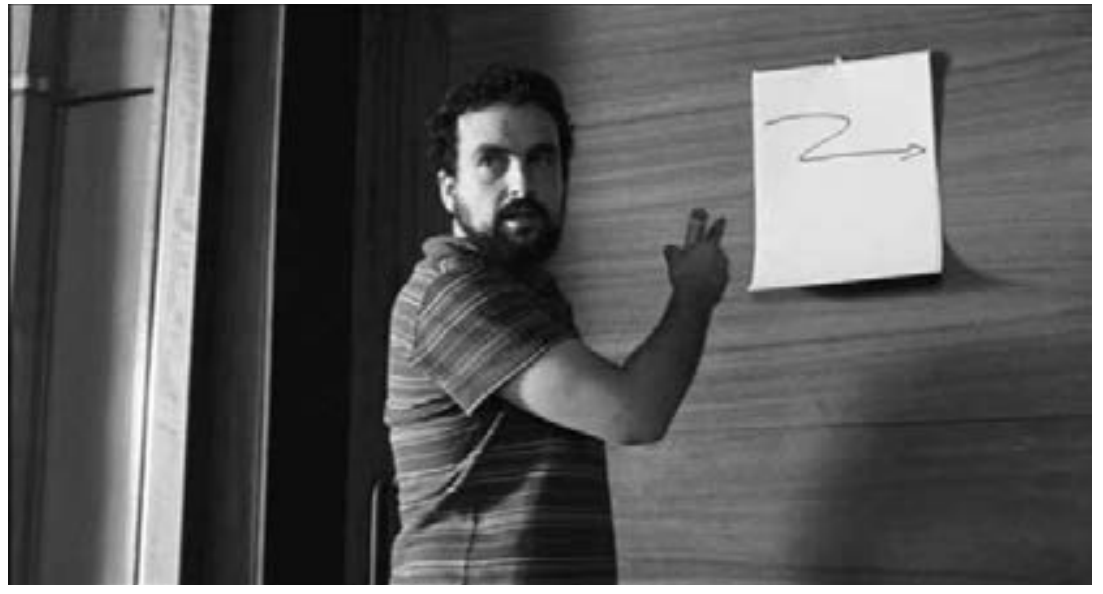

Fig. 4.4: A quick sketch that reveals the simple idea behind a complex film experience in Nacho Vigalondo's TIMECRIMES (2007), drawn by El Joven (played by Vigalondo himself). 


\section{Game Logic and the Fascination with Failure}

Another hypothesis to explain the popularity of contemporary puzzle films can be sought in the comparison between their viewing experiences and the logic of videogames. According to Jason Mittell, many contemporary complex narratives:

require the audience to learn the particular rules of a film to comprehend its narrative; movies like The Sixth Sense, Pulp Fiction, Memento, The Usual Suspects, Adaptation, Eternal Sunshine of the SPotless Mind, and Run Lola Run have all embraced a game aesthetic, inviting audiences to play along with the creators to crack the interpretive codes to make sense of their complex narrative strategies. But crucially, the goal of these puzzle films is not to solve the mysteries ahead of time; rather, we want to be competent enough to follow their narrative strategies but still relish in the pleasures of being manipulated successfully. $(2006,37-38)$

Similarly, Elliot Panek notes that:

An element of non-filmic interactive storytelling exists in these [puzzle] films. Younger audiences that are increasingly comfortable with the burgeoning interactive medium of video games may find puzzle narratives appealing for this reason. It is not enough to say that these characters are mentally unstable and that when the narration diverges from the classical mode, it is merely reflecting their fractured look on life. We seem to seek the nature of the instability even when we realize we are watching a psychological puzzle film, and take pleasure in trying to figure out the rules of the narration that presents the story to us. $(2006,87)$

According to Warren Buckland, the narrative logic of contemporary puzzle films can be traced to the emerging logic of new media, specifically of videogames (see his analysis of Duncan Jones's 2011 film SOURCE CODE in Buckland 2014b, 185-197). For him, the influence can be observed in puzzle films' promise of "reliable rules" - a characteristic that is central to the logic of videogames (Gottschalk 1995):

These rules, which are reliable in that they are systematic and unambiguous [...] constitute the video game's environment, or location, which is not restrained by the laws of the physical world. The game user can experience video pleasure primarily by attempting to master these rules - that is, 
decipher the game's logic. Moreover, the desire to attain mastery makes video games addictive, which at times can lead to the user's total absorption into the game's rules and environment. (Buckland 2014b, 187)

Although we believe a fundamental caution should be maintained with regard to claims crossing over from different media (film is, after all, still a noninteractive medium according to most definitions of interactivity), Mittell's, Panek's and Buckland's observations offer an interesting angle. Indeed, we would agree that in highly complex films, viewers do not simply experience complexity and dissonance, but are also often inclined to try to understand the underlying logic thereof - in Panek's words, to "seek the nature of the instability." In many cases, this does indeed involve attempts to discern a set of logical rules in the narration - rules that the viewer could ultimately master. However, as previously noted, impossible puzzle films do not seem to offer the "reward" usually associated either with puzzle films or with games (in the forms of a revealing twist, resolution or outcome, or in the reaching of a new level). Some films do not simply delay the viewer's access to the rules and logic that govern their narration, but sometimes even fully deny viewers such logic. Nonetheless, this does not need to make the game-logic analogy invalid for these films. There are two reasons for this. Firstly, we observed that complex stories such as impossible puzzle films often seem designed to keep viewers inclined to search for a logic to their stories, employing various (post)classical storytelling strategies that encourage such "classical" narrative engagement (Kiss and Willemsen 2017, 163-182). Viewers may therefore still find in these films the "promise of reliable rules" that Gottschalk and Buckland observe in games and cinematic puzzles. Secondly, it seems that failure forms an intrinsic, even pleasurable part of any gaming activity. As impossible puzzle films often evoke in viewers unsuccessful attempts to grasp their stories and story logic, a certain sense of "failure" also seems to characterize their experiences. An explanation for the appeal of such viewing effects could be found in humans' seemingly paradoxical fascination with failure. Regarding impossible fictional worlds, Umberto Eco already identified such appeal as "the pleasure of our logical and perceptual defeat" $([1990] 1994,77)$. But what is pleasurable about a cognitive and perceptual defeat? In his book on videogames (tellingly titled The Art of Failure), Danish ludologist, Jesper Juul, points out the initially somewhat counterintuitive fact that "players prefer games in which they fail" $(2013,2)$. Drawing from his own experience, Juul notes that "I dislike failing in games, but I dislike not failing even more" (2). By means of some elegantly simple experiments, Juul demonstrates the importance of failure 
and feelings of inadequacy in the context of videogames. He observes that "players who completed the game without failing gave it a lower rating than those who failed at least once" (35), and that "players rated the game significantly higher when they felt responsible for failure than when they did not" (53-54).

Juul's observations seem to rhyme with the psychological workings of impossible puzzle films. Comparable to how a game "promises us that we can remedy the problem if we keep playing" (7), impossible puzzle films may beguile viewers with a similar promise, as their highly complex (but seemingly logical) narration continuously encourages viewers to rationalize and narrativize the illogical. The prospect of the potential intelligibility of these films inspires viewers to keep trying to overcome their felt inadequacy - which, as Juul notes with regard to games, is "an inadequacy that they produce in us in the first place" (7). By arousing a sense of inadequacy, impossible puzzle films seem to trigger a similar motivational bias: viewers may feel that their competence or intelligence is being challenged in cracking the puzzle, and therefore give in to the urge to overcome "their" failure through recurring attempts at problem solving. To capture this recurring aspect of the process in gaming, Juul $(2013,60)$ introduces a model of the failureimprovement cycle of videogame play. The cycle consists of four steps: (1) a new goal is introduced; (2) failure presents the player as inadequate; (3) the player searches for the cause of the failure and improves; and (4) the player is no longer inadequate; he or she has new skills. A similar mechanism seems to be active in impossible puzzle film viewing, with the key difference being that the required "improvement" may not be satisfyingly reached. Rather, viewers' ongoing lack of understanding and constant feeling of inadequacy may become a driving force that keeps them invested in comprehending the story, and, eventually, might contribute to their evaluating the experience as engaging. In sum, this hypothesis assumes that the engaging potential of impossible puzzle films is partly managed by strategies that continuously challenge viewers' feeling of competence, which can contribute to the framing of the failure in achieving full comprehension as a fascinating experience.

\section{Effort Justification}

Related to our fascination with failure, another possible reason for the attraction of confusing and cognitively demanding narrative experiences could be sought in the psychological principle of effort justification. In social 
psychology, effort justification is understood as an everyday cognitive dissonance-reduction strategy - a mode of changing the value of existing cognitions. Simply put, the principle states that people tend to evaluate an outcome, reached goal, or completed task as being more valuable when this outcome has cost them more effort to achieve. It has been suggested that this principle is active in many different social and behavioral patterns. It can, for example, help to explain phenomena such as hazing and initiation rituals: by having to go through hardships or having to make an effort to be allowed into a social group, an individual is likely to value this membership more highly, as he or she has to justify the effort made (attaching a higher value to the outcome reduces the dissonance with regard to the more unpleasant aspects of the experience). Drawing on Leon Festinger's original theory of cognitive dissonance (1957), a classic study by Elliot Aronson and Judson Mills (1959) connected varying amounts of effort to evaluative judgments. Aronson and Mills hypothesized that the effort justification mechanism could be effective in any basic set of conditions regarding effort and evaluation: "For example, one would expect persons who travel a great distance to see a motion picture to be more impressed with it than those who see the same picture at a neighborhood theater" $(1959,177)$.

Cognitive scientist, Jim Davies (2014), extends the principle of effort justification to the realm of meaning-making. For him, discerned meaning becomes more valuable if it is attained through substantial cognitive effort. According to Davies, the pleasure of puzzles can also be related to this principle; after all, "[w]ith puzzles, the audience gets to appreciate so many things: the initial incongruity, the pleasure of knowing the solution, the pride of having discovered it themselves, and an increased value of the found solution due to idea effort justification" $(2014,143)$.

But how does this translate to an impossible puzzle? What is the mental payoff of the perpetually challenging experience that impossible puzzle films sometimes provide? It is apparent that the narrative comprehension of these films demands significantly more cognitive efforts than most classical stories or "ordinary" puzzle films (which provide or allow a relatively easy access to a coherent and logical solution to their conundrum). As elsewhere noted (Kiss and Willemsen 2017, 104-139), impossible puzzle films allow cognitive operations and interpretive strategies that can compensate for viewers' fruitless efforts to find a coherent and logical solution. We would therefore hypothesize that Aronson and Mills's "suffering-leading-to-liking" thesis (Gerard and Mathewson 1966) can play a role in the appreciation of more pervasively complex films as well: attributing a positive judgment to these films' rich affordances might tame the experienced dissonance with 
regard to the effort made. Simply put, one could presume that the general principle of effort justification still holds true for films that do not necessarily offer narrative closure or a satisfying resolution. According to this, the appeal of impossible puzzle films may stem from these films' offered analytical and interpretive richness, the intensified inspiration for forensic activities their puzzles call forth, and from viewers' general respect for a highly challenging experience that seems to outsmart them. These hypotheses could make for an interesting subject in terms of further empirical investigations.

\section{Diegetization of Decoupling}

According to cultural cognitivist, Barend van Heusden, the appeal of cognitively dissonant narrative art comes from the amplification of a very general human disposition - one that characterizes practically all our reallife and mediated narrative experiences (Van Heusden 2009; and personal correspondence). He reasons that cognitively dissonant scenarios make us reexperience the act of decoupling, which is not only an integral part of our cognition but also a core aspect of the general human condition.

As Merlin Donald has argued (1991, 2006), through the evolutionarily increased capacities of working memory, humans have become capable of decoupling memory from actuality:

Donald equates the origins of modern humans to a transition from episodic to mimetic cultures, or the transition from lives that are bounded to the immediacy of experience to lives that are lived not only in the present but also in the simulation or representation of this experience. (Rochat [2001] 2004, 73)

In this sense, the act of decoupling is the source of human imagination: being able to "decouple" from the actuality of our here-and-now experience enables us to simulate, represent, or even fantasize about alternative versions of our reality. Following this train of thought, decoupling allows mimesis, whereas "art is an inevitable by-product of mimesis" (Donald 2006, 14). Hence, as a result of the cognitive evolution of the human species and its developed capacity for decoupling, the nature of culture and the experience of mimetic art fundamentally bear elements of dissonance. This means that there is a fundamental, deep-seated (yet unconscious and rarely reflected) conflict between our actual and imagined experience: between the "here and now" of actual perception (the reality context of reading or viewing, 
that is, our reality as real readers and viewers) and the "there and then" virtual domain of narrative immersion (the diegetic world and its fictive population, which form the destination of our absorption and embodied identification). This "cognitive dissonance" is a result of the transfer from our real-life existence to the mediated art experience. ${ }^{8}$

If art is the mimetic imitation of an experience through representation by mediated simulation, then "metarepresentation" is a reflection on art's mimetic representation. Certain metarepresentational cinematic strategies may highlight the cognitive dissonance inherent to the experience of artistic representation. Films can thematize and manifest the act of decoupling through narrative diegetization of this very fundamental dissonance. A notable example of this is provided by the abundance of character duplications in impossible puzzle films. Character splitting, doubling, and multiplication provide powerful instances of the diegetization of decoupling's inherent dissonance. Looping narratives' character multiplications - such as in Roman Polanski's The Tenant (1976), Smith's Triangle, Vigalondo's Timecrimes, the Spierig brothers' Predestination (2014), or Lynch's Lost HIGHWAY - allow us to literally reexperience the underlying dissonance between our double presence of "here and now" and immersed "being there," which can be seen as a subtle addition to these films' attractiveness (beyond, and in case of Polanski's film, prior to the more obvious effects of digital lossless copying, video games' multiple lives, social media avatars, and other distinctly contemporary reasons that scholars and critics have attributed to the character-doubling "trend"). 9

\section{Fascination with Infinity}

Certain impossible puzzle films owe part of their attraction to the arousal of what seems to be a deeply rooted human fascination with infinity. Whether encountered via mathematics or geometry, cosmology, or theology, the idea of endlessness seems to exert a strong curiosity, detectable throughout Western cultural history and the arts (Maor 1987). Like mathematicians, visual artists have repeatedly attempted to capture infinity in an aesthetic form, for instance through endlessly looping patterns (comparable to the famous steps by Lionel and Roger Penrose [1958] - Fig. 4.5) or recursive mise-en-abymes (a picture of a picture in a picture in a picture - suggesting multiplication ad infinitum). Some impossible puzzle films similarly suggest "infinity," presenting narrative versions of infinite loops (for example, TRIANGLE and TimECRIMES) or endless narrative mise-en-abymes through 


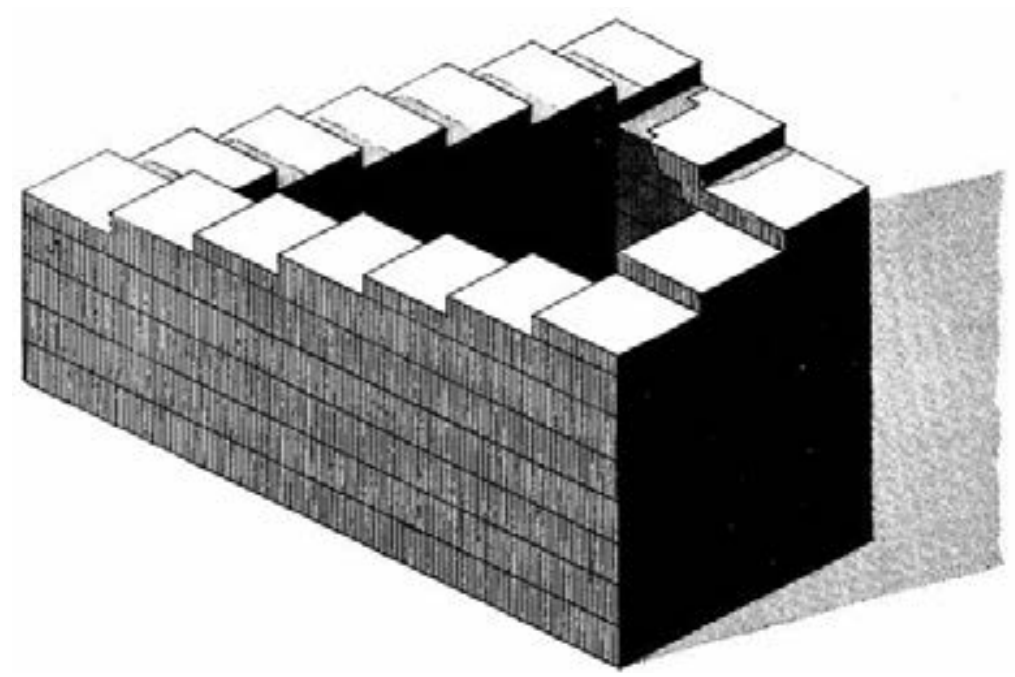

Fig. 4.5: The impossible loop of Penrose Steps by Lionel and Roger Penrose (1958).

embedded metalepses (for example, REALITY and SynecDoche, NEw YoRK, 2008). These peculiar "endless" narrative structures seem to exert a curious fascination.

Why is it that pondering "the infinite" is prone to evoking reactions of wonder or bewilderment? In a 1994 paper, psychologist, Ruma Falk, discusses how infinity seems to be "infinitely challenging to the human mind" (35). She notes that "people's intellectual attempts to cope with the puzzles posed by the infinite have been interwoven with a wide spectrum of emotional responses" (35). According to her, these emotions and fascinations are essentially triggered by the human inability to cope with the "disturbing contradictions" that endlessness entails (36). This inability, Falk argues, is grounded in two particular cognitive moves - neither of which is compatible with our habitual strategies of reasoning. Firstly, in order to grasp infinity, one needs to practice "the ability to suppress our imagination, at least the visual part of it" (54). This entails a conscious detachment from everyday experience and knowledge, common sense and the habitual formation of mental imagery, all of which imply (and depend upon) finiteness in the world around us. Therefore, coming to terms with infinity demands the challenge of "unlearning of old truisms" about the laws and dimensions of the world in which we live (53). Secondly, according to Falk, the infinite will always remain an abstraction - a concept that is beyond the reach of human experience and intuition, and that is best explained by scientific conceptualization. Like quantum mechanics, infinity proves very difficult to comprehend in 
terms of the realm of everyday experience, yet its workings can ultimately be understood through (scientific) argumentation and description. Falk illustrates this by referring to examples of so-called "super-task problems," borrowed from mathematical and psychological experiments: she asserts that as long as one tries to reconcile puzzles about the infinite rationally and commonsensically, they will elicit "bizarre conclusions" (55). Hence, Falk argues:

No real-life experiment can ever model the infinite. [...] Paradoxically, one needs a kind of (non-visual) vision that can accept the unimaginable. The key to abstract thought is its detachment, not only from sensory perception, but even from imagery. Dissociation from familiar aspects of reality and from strongly held beliefs may enable human understanding to surpass intuition. $(1994,37,54)$

Arguably, infinity derives its fascinating aesthetic potential from this challenging of familiar aspects of our (beliefs about) reality. Illustrations such as the Penroses' infinite steps or Escher's paradox loops (such as his 1959-196o lithograph Ascending and Descending) are examples of attempts "to capture infinity in a 'closed' composition" (Schattschneider [1990] 2005, 241). ${ }^{10}$ We have compared the narrative structures of impossible puzzle films to depictions such as Escher's (Kiss and Willemsen 2017, 86-91), noting how these films also make the seemingly impossible perceptually and conceptually available. Some impossible puzzle films (not all, it must be noted) play with narrative mechanisms that suggest "infinite" outcomes. Films such as TRIANGLE or THE TENANT present stories that turn into endless loops without beginnings or endings; REALITY constructs a mise-en-abyme in which different story levels are contained in one another, offering a continuous paradox; and SyNeCDOCHE, New YoRK plays with another kind of mise-en-abyme, one that is implied through a constantly duplicating simulacrum: as protagonist Caden wants to direct a play that honestly and realistically captures his real, mundane life, he finds that his play must also include him making the play, which then needs to include a play about him making that play - a logic that ultimately points toward a potentially infinite recursion of plays within plays within plays.

These examples all use circular structures and recursive multiplications as narrative devices to suggest endlessly looping or duplicating diegetic realities. Although such storyworlds exert a strong sense of "impossibility," they are, at the same time, presented as coherent, "inhabitable" and, up to a point, imaginable. In their totality, however, potentially "infinite" story patterns 
such as these indeed entail, as Falk noted, "disturbing contradictions" - or, we would say, "dissonant cognitions": they clash with common sense, reason, and everyday experiential evidence. It seems that the challenge of coming to terms with the infinite pushes the limits of our embodied and situated cognition - which is not surprising, considering that our cognition emerges in, and is directed at, a seemingly "finite" world (as our lived environment is characterized by apparent physical boundaries and limitations). The way in which infinity surpasses these everyday intuitions and defies our imagination may therefore be experienced as engaging, uncanny, enthralling, or simply surprising. Some impossible puzzle films play on this effect, suggesting infinity to further fuel the fascination that viewers find in the narrative acrobatics and cognitive challenge of metalepses, loops, and other intricately multiplying story patterns.

\section{Destabilized Ontological Certainties}

Metalepses in narrative fiction collapse fictional boundaries among embedded narrative frames. While our fascination with infinity can be triggered by simulating the possibility of endless multiplication of embedded levels in a story (that is, stories within stories), metaleptic transgressions work by breaking the boundaries between story levels, often playing with the odd option of extending the fictional to the real (for example, real writers appearing in their fictional stories). Complex films and impossible puzzle films, we hypothesize, often seem to use such "ontological metalepses" to arouse uncanny, potentially intriguing emotional and intellectual effects.

Contemporary complex films frequently employ ontological metalepses to present fictional transgressions between their diegetic and embedded hypodiegetic story levels. Examples may include Marc Forster's STRANGER THAN Fiction (2006), in which Harold Crick (Will Ferrell) becomes aware that he is a fictional character in a still-developing book of an author, with whom he even shares the narrative level; or Spike Jonze's ADAPTATION (2002), where the film's real screenplay writer, Charlie Kaufman, writes himself into his film script, which becomes the film that the viewer is watching. A compelling literary case is provided by Julio Cortázar's 1962 short story The Continuity of Parks, wherein the protagonist appears to be threatened by a character from a book he is reading.

Due to the logic that such porous narrative structures allow, ontological metalepses may have the potential to awaken in readers or viewers a certain "sense of logical unease" (Eco 1979, 234). Stories such as Cortázar's collapse 
very basic ontological boundaries - not only between different story layers, but also between fictional and real-life levels of the experienced fiction and experiencing context. As for the latter, the "ontologically threatening" potential of metalepsis was acknowledged already in the theorizing of Gérard Genette ([1972] 1980, 236). Genette quoted Jorge Luis Borges - himself a master of narrative metalepses - who, being fascinated by such deep ontological uncertainties, noted that "if the characters in a story can be readers or viewers, then we, their readers or spectators, can be fictitious" ([1960] 1964, 46). According to this, an ontological metalepsis might "amount to a double catharsis, a representational and an existential one" (Meister 2003; our emphases).

Impossible puzzle films, with their tangled complexity and ambiguous hierarchies among different levels and multiple plots, are especially prone to arousing a certain ontological uncertainty in viewers. These films frequently play with vague or transgressed boundaries between dream and reality, fiction and real life, or telling and told. In a rare but registered effect, ontological metalepses might even lead to a psychologically identified disorder that is tellingly called the Truman Syndrome (Fusar-Poli et al. 2008), in which the patient suffers from a delusion that his or her life is part of a fictional story, staged as a play or reality show and controlled by unseen powers. ${ }^{11}$ Although we would not want to claim that impossible puzzle films' narrative tactics instill such degrees of psychological (truly existential) anxiety in their viewers, it is reasonable to assert that some playful metalepses are able to set in motion the idea (and subsequent feelings) of ontological uncertainty, adding to the fascination and perhaps appreciation of their ambiguous, paradoxical, and dissonant experience while maintaining their stories' stubborn mysteries.

Take, for instance, ADAPTATION's playful destabilization of its viewers' ontological positions and assumptions. The story revolves around a fictionalized version of the actual screenplay writer, Charlie Kaufman. It shows Kaufman's (Nicolas Cage) struggle to adapt a book, and his decision to write a film about this struggle, which turns out to be the film we are watching. Director, Jonze, and screenwriter, Kaufman, not only play with these transgressions within the film's narrative levels (writer/written), but further utilize the destabilizing potential of their metalepsis by allowing the fiction to "leak" into the film's paratextual and actual contexts: for example, ADAPTATION's credits mention Charlie Kaufman's fictional brother from the film, Donald Kaufman (also played by Nicolas Cage), as a cowriter of the film's real screenplay. ${ }^{12}$ Also, the film further plays with its own reality status by including scenes about the making of Jonze and Kaufman's previous 
movie, Being John MALKOviCH (1999), as part of AdAPTATiOn's fiction. Such strategies not only obfuscate the relation between the adapted and adaptation in the film, but also undercut viewers' "uppermost" controlling position regarding the fictional and the real. This involvement of the viewer through an ultimate metaleptic pop can be seen as a device that heightens this kind of cinema's engaging capacity, making viewers part of the film's complex game.

\section{Eudaimonic Motivations and Intrinsic Needs}

Above, we characterized the attractiveness of confusing stories as somewhat "paradoxical." Similar to the well-known sadness-paradox in art and media studies which says that people willingly engage with artworks that evoke negative emotions, such as sadness, that they would normally avoid, one encounters what resembles a "confusion-paradox": it seems that in narrative art and fiction, the negative valence of being confused can be considered enjoyable. However, as we hope to have demonstrated, highly complex movies are also capable of engaging and fascinating viewers in a variety of ways. How, then, should this "paradox of the confusion-paradox" be resolved?

One way of escaping the confusion-paradox is by emphasizing the so-called eudaimonic motivations that viewers may have for engaging with fiction. In addressing the issue of negative emotions in art, media psychologists, Mary Beth Oliver and Arthur A. Raney, have argued that "people consume media entertainment in the pursuit of pleasure and amusement (hedonic motivations) and as part of their general need to search for and ponder life's meaning, truths, and purposes - motivations that we characterize as 'eudaimonic" (2011, 985; our emphases). Indeed, the attraction to highly complex stories becomes less paradoxical if one drops the (arguably erroneous) assumption that the engagement with fiction should be conceived of as only "hedonically" motivated - that is, strictly in terms of bringing "entertaining pleasure." Most of the hypotheses developed in this chapter concern cognitive and interpretive reflections that are better characterized as driven by eudaimonic motivations (reflection, truth-seeking, or self-development) than as strictly hedonically motivated. However, postulating a distinction between "hedonic" and "eudaimonic" drives still implies a basic difference between "fun" and "meaningful" experiences that seems problematic. After all, can hedonic pleasures not be found in the gratification of eudaimonic concerns as well?

Having the same dilemma, Ron Tamborini and his colleagues (2010) suggested that it would be better to approach eudaimonic motivations for 
media consumption in relation to the satisfaction of people's intrinsic needs. In conceptualizing these "intrinsic needs," the researchers used Edward L. Deci and Richard M. Ryan's self-determination theory (1985). This seminal theoretical model assumes three basic psychological needs in individuals, namely autonomy, competence, and relatedness:

autonomy [is] a sense of volition or willingness when doing a task (Deci and Ryan 200o); competence [is] a need for challenge and feelings of effectance (Deci 1975); and relatedness [is] a need to feel connected with others (Ryan and Deci 2001). (Tamborini et al. 2010, 761)

While working with experiments involving a videogame, the researchers confirmed the role of these needs in relation to media consumption. Yet they also noted that there is "no basis to believe that our definition of enjoyment as the satisfaction of needs is limited to video games" (771). Therefore, our final hypothesis concerns the enjoyment and engagement of impossible puzzle films in light of these intrinsic psychological needs. We would suggest that the attractiveness of complex films should not be seen as strictly hedonic (pleasurable or entertaining) or merely eudaimonic reflections (pondering life's complexities or achieving personal development), but should be understood as appealing to viewers' psychological intrinsic needs. For some people, impossible puzzle films may resonate with their need for autonomy (as the interpretive freedom and playfulness of these films leave a relatively high amount of choice and authority to the individual viewer), or may be a means to establish relatedness (for instance, through collective forensic fan activities, or the social rewards of sharing of interpretations, plot maps, or explanatory videos online). Yet the key concept in terms of the enjoyment of impossible puzzle films seems to lie in the notion of competence. On the basis of the above hypotheses and arguments, we contend that highly complex films - by challenging and entertaining a variety of cognitive, analytical, and interpretive skills - engage viewers by appealing to their intrinsic need for competence and effectance. ${ }^{13}$ Whether it is about finding an interpretation that works, grasping a story's intricate mechanisms, dealing with ontological uncertainties, or mapping a plot, enjoying these films usually entails engaging in simulated challenges that playfully (and safely) address viewers' need to feel competent and skilled. ${ }^{14}$ As Jason Mittell noted, viewers of complex narratives "relish in the pleasures of being manipulated" but, ultimately, "want to be competent enough to follow their narrative strategies" $(2006,38)$. The urge to "keep up" with a complex story arguably tickles viewers' self-esteem and engages their potential for effectance. 
In conclusion to our study, we would propose that feeling "challenged" by complex movies may be more important than solving their puzzles. In this light, the success of impossible puzzle films can be seen as the result of a narrative audacity that takes its viewers' "empowered" positions into consideration; these films dare to enduringly confuse viewers, and boldly leave large chunks of the interpretive and analytical work up to their cognitive and interpretive competences. The narrative and psychological pressures on viewers to resolve dissonances and achieve comprehension make room for all kinds of creative, intellectual, analytical, and interpretive skills and processes. This, especially in a mainstream context, is quite novel, but, as the trend proves, not inconceivable. Surely, our proposition presupposes viewers' resonance with this kind of cinema, and entails that varying degrees of competency (in terms of film and media literacy) will form a key factor in terms of their varying enjoyment of such highly complex films. In this sense, impossible puzzle films may be seen as the product of a specific moment in our media- and narrative-saturated time. Films such as these are able to cognitively challenge and intellectually intrigue a number of viewers who may have already grown accustomed to ever-increasing amounts and forms of mediacy, narratives, and complication - whether in popular fiction or in culture at large. Cinematic versions of impossible puzzles thereby seem to reflect the larger cultural shifts behind their emergence: not only do they appeal to a deep-seated human hunger to solve puzzles, they also embrace our life's complexities, providing enigmatic journeys into the impossible.

\section{Notes}

1. This contribution is an edited excerpt from the final chapter of the monograph Impossible Puzzle Films: A Cognitive Approach to Contemporary Complex Cinema (Kiss and Willemsen 2017, 183-207). We wish to express our gratitude to Edinburgh University Press for granting us permission to use this reprint.

2. This use of "cognitive dissonance" is not to be confused with this term's established sociopsychological meaning, which refers to the effect of inconsistencies in an individual's behaviors or beliefs in real-world situations (e.g., Cooper 2007; Stone 1999) and has also been used to describe, for example attitudes toward fictional characters or situations (e.g., Caracciolo 2013; Van der Pol 2013). Although our use of the term "cognitive dissonance" in narrative comprehension shares some similarities with the cognitive core of Leon Festinger's original theory $(1957,31,13)$ and its suggestion of how dissonances between cognitions elicit a pressure to resolve or deal with 
the conflict, there are also significant differences (e.g., between fictional and real-world situations, or between values and logical beliefs). A more elaborate discussion of these differences and overlaps was included in our original study (Kiss and Willemsen 2017, 67-70).

3. See also Nitzan Ben Shaul's analysis (2012) of how many classical narrative films induce a certain "close-mindedness" in viewers, whereas some films do allow them the distinct pleasure of entertaining their ability for "optional thinking," for instance by offering alternative narrative paths among which viewers can choose or imagine different possibilities.

4. For instance, cultural philosopher, Thijs Lijster (2014), proposes such a view on the historical development of the detective/mystery genre. According to Lijster, the detective fiction evolved from the celebration of Enlightenment values and scientific reason (cf. Sherlock Holmes's ever-successful use of deductive logic and inference-making) to a genre riddled with paranoia, labyrinth-like enigmas and mysteries that can no longer be solved or understood by a single detective (cf. InHEREnT Vice, Paul Thomas Anderson, 2014). Moreover, the detectives themselves became increasingly unreliable, questionable, and flawed throughout twentieth-century fiction. For Lijster, these shifts mirror the state of the (post)modern condition from which the stories originate, such as the increasing cultural complexity and socioeconomic decentralization of our times.

5. $\quad$ For theoretical arguments (Johnson [1987] 1990; Slors 1998; Menary 2008) and neuroscientific proofs (Gallese and Lakoff 2005), consult the previously published article (Kiss 2013).

6. As for such topographic mapping, because "[p]eople read for the plot and not for the map" (Ryan 2003, 238), it can be said that both film viewers and "readers of print texts rarely maintain an 'accurate map of spatial relations' in the represented storyworld" (Ciccoricco 2007, 54). It is obvious that the topographic practice of literary or visual cartography is a useful tool for creative artists, but it is rarely triggered as a "natural" reader or viewer response. Yet there is empirical proof that adult viewers encode a more or less stable spatial layout "even when there is no explicit demand for them to do so" (Levin and Wang 2009, 26).

7. The method of graphical extension of mental mapping might be implemented in the creative practice of designing narrative experiences as well. For instance, Christopher Nolan is known for making such sketches, as revealed in the shooting script for his fairly complex film INCEPTION (2010).

8. In Van Heusden's words, since “[w]e do not live in, and reality does not coincide with, our representations" $(2009,614)$, the possible awareness of the fundamental difference between our experiential domains of "here and now" reality and "there and then" simulation of this reality "seems to be basic to human cognition" (614).

9. Beyond technology-fuelled allegories, character-duplication films such as ENEMY "[tap] into the root of our newfound doppelgänger obsession and 
fear. Many of us are afraid that we're simply not enough as we are - that we're not cool enough, pretty enough, passionate enough, or interesting enough" (Wilkinson 2014).

10. Penrose and Penrose's article in the British Journal of Psychology (1958) featured the impossible staircase, which then, in fact, inspired Escher's Ascending and Descending (1960).

11. The term stems from the story of Truman Burbank, who unknowingly participates in a reality television program in Peter Weir's The Truman SHow (1998).

12. This even resulted in an Oscar nomination for "Charlie Kaufman and Donald Kaufman" (for Best Adapted Screenplay), making Donald the first ever entirely fictitious Oscar nominee.

13. Effectance is defined in organisational psychology as "the causal effect of an object in the environment" (Nugent, Pam M.S., "EFFECTANCE," PsychologyDictionary.org, April 7, 2013).

14. Of course, formally complex stories are not the only types of fiction that play on this. For instance, in his 1991 model of mystery enjoyment, Dolf Zillmann argued for the role of competence in all mystery fiction, noting that "the enjoyment of certain forms of mystery is motivated by self-esteem needs akin to competence" (Tamborini et al. 2010, 771). Although impossible puzzle films do not offer coherent and explicit answers that much of mystery fiction requires and provides (such as a clear answer to the "whodunit" question in detective stories), they do seem to tease a similar viewing disposition.

\section{References and Further Reading}

Alber, Jan. 2016. Unnatural Narrative: Impossible Worlds in Fiction and Drama. Lincoln, NE: University of Nebraska Press.

Ang, Ien. 2011. "Navigating Complexity: From Cultural Critique to Cultural Intelligence." Continuum 25, no. 6: 779-794.

Aronson, Elliot, and Judson Mills. 1959. "The Effect of Severity of Initiation on Liking for a Group." Journal of Abnormal and Social Psychology 59, no. 2 (September): 177-181.

Ben Shaul, Nitzan. 2012. Cinema of Choice: Optional Thinking and Narrative Movies. New York and Oxford: Berghahn.

Bordwell, David. 1985. Narration in the Fiction Film. Madison, WI: University of Wisconsin Press. Borges, Jorge Luis. 1964 [1960]. Other Inquisitions 1937-1952. Austin, TX: University of Texas Press.

Buckland, Warren. 2009. Puzzle Films: Complex Storytelling in Contemporary Cinema. Oxford: Wiley-Blackwell.

-. 2014a. Hollywood Puzzle Films. London: Routledge.

—. 2014b. “Source Code's Video Game Logic." In Hollywood Puzzle Films, edited by Warren Buckland, 185-197. London: Routledge.

Cameron, Allan. 2008. Modular Narratives in Contemporary Cinema. Basingstoke: Palgrave Macmillan. 
Campora, Matthew. 2014. Subjective Realist Cinema: From Expressionism to Inception. New York and Oxford: Berghahn.

Caracciolo, Marco. 2013. "Patterns of Cognitive Dissonance in Readers' Engagement with Characters." Enthymema 8: 21-37.

Ciccoricco, David. 2007. Reading Network Fiction. Tuscaloosa, AL: University of Alabama Press.

Coëgnarts, Maarten, Miklós Kiss, Peter Kravanja, and Steven Willemsen. 2016. "Seeing Yourself in the Past: The Role of Situational (Dis)continuity and Conceptual Metaphor in the Understanding of Complex Cases of Character Perception." Projections 10, no. 1 (June): 114-138.

Cooper, Joel. 2007. Cognitive Dissonance. Fifty Years of Classic Theory. Los Angeles: Sage.

Cortázar, Julio. 2014 [1962]. "Continuity of Parks." In Hopscotch; Blow-Up; We Love Glenda So Much, translated by Gregory Rabassa and Paul Blackburn, 625-626. New York, London, Toronto: Everyman's Library.

Davies, Jim. 2014. Riveted: The Science of Why Jokes Make Us Laugh, Movies Make Us Cry, and Religion Makes Us Feel One with the Universe. New York: Palgrave Macmillan.

Deci, Edward L. 1975. Intrinsic Motivation. New York: Plenum Press.

Deci, Edward L., and Richard M. Ryan. 1985. Intrinsic Motivation and Self-Determination in Human Behaviour. New York: Plenum.

-. 2000. "The 'What' and 'Why' of Goal Pursuits: Human Needs and the Self-Determination of Behavior." Psychological Inquiry 11, no. 4 (October): 227-268.

Donald, Merlin. 1991. Origins of the Modern Mind: Three Stages in the Evolution of Culture and Cognition. Cambridge, MA: Harvard University Press.

-. 2006. "Art and Cognitive Evolution." In The Artful Mind. Cognitive Science and the Riddle of Human Creativity, edited by Mark Turner, 3-20. New York: Oxford University Press.

Eco, Umberto. 1979. The Role of the Reader: Explorations in the Semiotics of Texts. Bloomington, IN: Indiana University Press.

—. 1994 [1990]. The Limits of Interpretation. Bloomington: Indiana University Press.

Elsaesser, Thomas. 2009. "The Mind-Game Film." In Puzzle Films: Complex Storytelling in Contemporary Cinema, edited by Warren Buckland, 13-41. Oxford: Wiley-Blackwell.

—. 2018. "Contingency, Causality, Complexity: Distributed Agency in the Mind-Game Film." New Review of Film and Television Studies 16, no. 1 (January):1-39. DOI:10.1080/17400309.2017.1411870.

Falk, Ruma. 1994. “Infinity: A Cognitive Challenge.” Theory \& Psychology 4, no. 1: 35-6o.

Festinger, Leon. 1957. A Theory of Cognitive Dissonance. Evanston, IL: Row.

Fusar-Poli, Paolo, Oliver Howes, Lucia Valmaggia, and Philip McGuire. 2008. "'Truman Signs' and Vulnerability to Psychosis." The British Journal of Psychiatry 193, no.2 (September): 167-169.

Gallese, Vittorio, and George Lakoff. 2005. "The Brain's Concepts: The Role of the Sensory-Motor System in Conceptual Knowledge." Cognitive Neuropsychology 21: 1-25.

Genette, Gérard. 1980 [1972]. Narrative Discourse. Ithaca, NY: Cornell University Press.

Gerard, Harold B., and Grover C. Mathewson. 1966. "The Effects of Severity of Initiation on Liking for a Group: A Replication." Journal of Experimental Social Psychology 2, no. 3 (July): 278-287.

Gottschalk, Simon. 1995. "Videology: Video-Games as Postmodern Sites/Sights of Ideological Reproduction." Symbolic Interaction 18, no. 1 (Spring): 1-18.

Heusden, Barend van. 2009. "Semiotic Cognition and the Logic of Culture." Pragmatics and Cognition 17, no. 3: 611-627.

Johnson, Mark. 1990 [1987]. The Body in the Mind. The Bodily Basis of Meaning, Imagination, and Reason. Chicago, IL and London: University of Chicago Press.

Juul, Jesper. 2013. The Art of Failure. An Essay on the Pain of Playing Video Games. Cambridge, MA: MIT Press.

Kiss, Miklós. 2012. "Narrative Metalepsis as Diegetic Concept in Christopher Nolan's Inception." Acta Film and Media Studies 5: 35-54. 
-. 2013. "Navigation in Complex Films: Real-life Embodied Experiences Underlying Narrative Categorisation." In (Dis)Orienting Media and Narrative Mazes, edited by Julia Eckel, Bernd Leiendecker, Daniela Olek, and Christine Piepiorka, 237-256. Bielefeld: Transcript.

-. 2015. "Film Narrative and Embodied Cognition: The Impact of Image Schemas on Narrative Form." In Embodied Cognition and Cinema, edited by Maarten Coëgnarts and Peter Kravanja, 43-61. Leuven: Leuven University Press.

Kiss, Miklós, and Steven Willemsen. 2017. Impossible Puzzle Films: A Cognitive Approach to Contemporary Complex Cinema. Edinburgh: Edinburgh University Press.

Klecker, Cornelia. 2013. "Mind-Tricking Narratives: Between Classical and Art-Cinema Narration." Poetics Today 34, no. 1-2 (Spring/Summer): 119-146.

Korthals Altes, Liesbeth. 2014. Ethos and Narrative Interpretation: The Negotiation of Values in Fiction. Lincoln, NE: University of Nebraska Press.

Levin, Daniel T., and Caryn Wang. 2009. "Spatial Representation in Cognitive Science and Film." Projections 3, no. 1: 24-52.

Lijster, Thijs. 2014. "Watching the Detectives." De Groene Amsterdammer, May 27, 2014. www. groene.nl/artikel/watching-the-detectives.

Maor, Eli. 1987. To Infinity and Beyond: A Cultural History of the Infinite. Princeton, NJ: Princeton University Press.

Meister, Jan Christoph. 2003. "The Metalepticon: A Computational Approach to Metalepsis." https:// www.icn.uni-hamburg.de/sites/default/files/download/publications/jcm-metalepticon.pdf.

Menary, Richard. 2008. "Embodied Narratives." Journal of Consciousness Studies 15, no. 6 (June): 63-84.

Mittell, Jason. 2006. "Narrative Complexity in Contemporary American Television." The Velvet Light Trap 58: 29-40.

-. 2015. Complex TV. The Poetics of Contemporary Television Storytelling. New York: New York University Press.

Oliver, Mary Beth, and Arthur A. Raney. 2011. "Entertainment as Pleasurable and Meaningful: Identifying Hedonic and Eudaimonic Motivations for Entertainment Consumption." Journal of Communication 61, no. 5: 984-1004.

Panek, Elliot. 2006. "The Poet and the Detective: Defining the Psychological Puzzle Film." Film Criticism 31, no. 1-2: 62-88.

Penrose, Lionel S., and Robert Penrose. 1958. "Impossible Objects: A Special Type of Visual Illusion." British Journal of Psychology 49, no. 1 (February): 31-33.

Pol, Gerwin van der. 2013. "Cognitive Dissonance as an Effect of Watching Amator." New Review of Film and Television Studies 11, no. 3: 354-373.

Rochat, Philippe. 2004 [2001]. The Infant's World. Cambridge, MA: Harvard University Press.

Ros, Vincent, and Miklós Kiss. 2018. "Disrupted PECMA Flows: A Cognitive Approach to the Experience of Narrative Complexity in Film." Projections (forthcoming).

Ryan, Marie-Laure. 2003. "Cognitive Maps and the Construction of Narrative Space." In Narrative Theory and the Cognitive Sciences, edited by David Herman, 214-242. Stanford, CA: CSLI.

Ryan, Richard M., and Edward L. Deci. 2001. "On Happiness and Human Potentials: A Review of Research on Hedonic and Eudaimonic Well-Being." In Annual Review of Psychology, edited by Susan T. Fiske, 141-166. Vol. 52. Palo Alto, CA: Annual Reviews.

Schattschneider, Doris. 2005 [1990]. M.C. Escher: Visions of Symmetry. London: Thames and Hudson.

Simons, Jan. 2008. "Complex Narratives." New Review of Film and Television Studies 6, no. 2:111-126.

Slors, Marc. 1998. "Two Concepts of Psychological Continuity." Philosophical Explorations 1, no. 1: 61-80. 
Staiger, Janet. 2006. “Complex Narratives: An Introduction.” Film Criticism 31, no. 1-2 (Fall/ Winter): $2-4$.

Stone, Jeff. 1999. "What Exactly Have I Done? The Role of Self-Attribute Accessibility in Dissonance." In Cognitive Dissonance: Progress on a Pivotal Theory in Social Psychology, edited by Eddie Harmon-Jones and Judson Mills, 175-201. Washington, DC: American Psychological Association.

Tamborini, Ron, Nicholas David Bowman, Allison Eden, Matthew Grizzard, and Ashley Organ. 2010. "Defining Media Enjoyment as the Satisfaction of Intrinsic Needs." Journal of Communication 6o, no. 4 (Fall): 758-777.

Tsur, Reuven. 1975. “Two Critical Attitudes: Quest for Certitude and Negative Capability.” College English 36, no. 7: 776-788.

Webster, Donna M., and Arie W. Kruglanski. 1994. "Individual Differences in Need for Cognitive Closure." Journal of Personality and Social Psychology 67, no. 6: 1049-1062.

Wilkinson, Alissa. 2014. "What's With All the Movies About Doppelgängers?" The Atlantic, March 14, 1994. www.theatlantic.com/entertainment/archive/2014/03/whats-with-all-themovies-about-doppelg-ngers/284413.

Willemsen, Steven. 2018. "The Cognitive and Hermeneutic Dynamics of Complex Film Narratives." Dissertation, Groningen: University of Groningen.

Willemsen, Steven, and Miklós Kiss. 2017. "Resistance to Narrative in Narrative Film: Excessive Complexity in Quentin Dupieux's Réalité (2014)." Global Media Journal: Australian Edition 11, no. 1. http://www.hca.westernsydney.edu.au/gmjau/?p=3081.

Zillmann, Dolf. 1991. "The Logic of Suspense and Mystery." In Responding to the Screen:Reception and Reaction Processes, edited by Jennings Bryant and Dolf Zillmann, 281-303. Hillsdale, NJ: Erlbaum. 